\title{
Balanço cátion-aniônico da dieta na composição do leite
}

\author{
Dietary cation anion balance on milk composition \\ Lisia Bertonha Correa $^{1}$ Marcus Antonio Zanetti ${ }^{2}$ Gustavo Ribeiro Del Claro ${ }^{3}$ \\ Fernanda Alves de Paiva ${ }^{3}$ Lucas Domenico Elmor ${ }^{4}$
}

\section{RESUMO}

Foram utilizadas oito vacas Holandesas em lactação, distribuídas em um quadrado latino $(4 \times 4)$, replicado, num experimento conduzido durante o verão, por um período de 72 dias. $O$ objetivo deste trabalho foi estudar quatro níveis de dietas catiônicas sobre a composição e as propriedades físico-químicas do leite em vacas após o pico de lactação. Para a manipulação do balanço cátion-aniônico da dieta $(B C A D)$, foram adicionadas diferentes concentrações de bicarbonato de sódio nas dietas, obtendo-se os seguintes tratamentos: $+150,+250,+400$ e $+500 \mathrm{mEq} \mathrm{kg-1} \mathrm{MS}$. Não houve diferença significativa para as variáveis: porcentagem de gordura, densidade e índice crioscópico do leite. $\mathrm{O} p \mathrm{pH}$ do leite aumentou linearmente e a acidez apresentou resposta cúbica com o aumento do BCAD.

Palavras-chave: ânions, bicarbonato de sódio, cátions, minerais, nutrição.

\section{ABSTRACT}

Eight lactating Holsteins cows were distributed in a $4 \times 4$ replicated Latin square in the summer for 72 days. The objective of this research was to study the effect of four levels of cationic diets on the composition and physic-chemistries parameters of milk in cows after the lactation peak. For dietary cation anion balance manipulation, different concentrations of the sodium bicarbonate were added in the diets and the following treatments were obtainned: $+150,+250,+400 e$ $+500 \mathrm{mEq} \mathrm{kg}^{-1} \mathrm{DM}$. The diets did not affect fat percentage and variables, density and crioscopic indice in the milk. Milk pH increased linearly and acidity decreased cubically with increased of dietary $C A B$.

Key words: anion, cation, sodium bicarbonate, minerals, nutrition.

\section{INTRODUÇÃO}

O balanço cátion-aniônico da dieta (BCAD) representa a diferença entre os cátions e os ânions presentes na dieta, podendo ser calculado em $\mathrm{mEq}$ de $(\mathrm{Na}+\mathrm{K})-(\mathrm{Cl}+\mathrm{S})$ por quilograma de matéria seca $(\mathrm{MS})$. A principal ação do BCAD é atuar na regulação do equilíbrio ácido-base do organismo. Nos últimos anos, muito se tem discutido a respeito da manipulação desse equilíbrio nos diferentes processos metabólicos dos animais, principalmente devido a sua estreita relação com o desempenho animal. Dietas catiônicas provocam alcalose metabólica, sendo denominadas dietas alcalinogênicas. As dietas aniônicas provocam uma acidose metabólica, sendo então acidogênicas (BLOCK, 1984). Dietas altamente catiônicas para vacas em lactação têm aumentado a ingestão de matéria seca (IMS) e a produção de leite, minimizando os efeitos do estresse pelo calor. Segundo SCHNEIDER et al. (1986), vacas em estresse calórico podem ter aumento na produção de leite devido ao consumo de bicarbonato de sódio, porém, nesse caso, esse aumento pode ser causado principalmente pela presença do sódio.

O BCAD também pode ter influência sobre a composição do leite. WATERMAN et al. (1991), em experimento com vacas no meio da lactação e dietas com níveis diferentes de BCAD, observaram que a alta concentração de cátions aumentou a porcentagem de gordura do leite. De acordo com ZIA et al. (2001), durante o pico da lactação, a produção de leite respondeu

\footnotetext{
${ }^{1}$ Departamento de Zootecnia, Faculdade de Zootecnia e Engenharia de Alimentos (FZEA), Universiade de São Paulo (USP), 13635-900, Pirassununga, SP, Brasil. E-mail:lisiabc@yahoo.com.br. Autor para correspondência.

${ }^{2}$ Departamento de Zootecnia, FZEA, USP, Pirassununga, SP, Brasil.

${ }^{3}$ FZEA, USP, Pirassununga, SP, Brasil.

${ }^{4}$ Curso de Zootecnia, FZEA, USP, Pirassununga, São Paulo, Brasil.
} 
positivamente ao maior BCAD (+20 comparado com $\left.10 \mathrm{mEq} \mathrm{g}^{-1}\right)$. As porcentagens de gordura e proteína do leite aumentaram com o aumento do BCAD. RAMANA et al. (2002), estudando o efeito da adição do bicarbonato de sódio na composição e na produção de leite em vacas cruzadas, encontraram aumento na gordura do leite; entretanto, não encontraram efeito significativo na produção e na porcentagem de proteína do leite. KENNELLY et al. (1999), trabalhando com vacas holandesas, dietas com diferentes relações volumoso:concentrado e inclusão de bicarbonato de sódio, observaram que a dieta de alto concentrado, mesmo com a adição do tampão, diminuiu a gordura do leite.

O objetivo deste trabalho foi estudar os efeitos de diferentes níveis de dieta catiônica $(+150$, $+250,+400,+500 \mathrm{mEq} \mathrm{kg}^{-1} \mathrm{MS}$ ), sobre a composição e os parâmetros físico-químicos do leite por meio da inclusão de bicarbonato de sódio em vacas após o pico de lactação .

\section{MATERIAL E MÉTODOS}

O experimento foi conduzido na Faculdade de Zootecnia e Engenharia de Alimentos, USP, Campus de Pirassununga, no período de dezembro de 2003 a fevereiro de 2004. Foram utilizadas oito vacas da raça Holandesa, multíparas, que se encontravam em período pós-pico de lactação. As vacas possuíam escore entre 3,0 e 3,5, peso entre 550 e $600 \mathrm{~kg}$ e produção média anterior de $6.700 \mathrm{~kg}$ lactação ${ }^{-1}$.
Foram oferecidos quatro tratamentos aos animais, diferindo nas concentrações de bicarbonato de sódio, de modo que as dietas apresentassem diferentes valores de BCAD. Esses valores foram calculados em mEq de $(\mathrm{Na}+\mathrm{K})-(\mathrm{Cl}+\mathrm{S}) \mathrm{kg}^{-1} \mathrm{MS}$. Dessa forma, os animais receberam quatro diferentes níveis de BCAD (+150; +250; +400 e +500mEq kg-1 MS). O tratamento com $+150 \mathrm{mEq} \mathrm{kg}{ }^{-1} \mathrm{MS}$ não tinha a adição de bicarbonato de sódio.

A dieta foi oferecida na forma de ração total, com $60 \%$ de concentrado e $40 \%$ de volumoso, formuladas com base nas recomendações do NRC (2001). A tabela 1 apresenta a proporção dos ingredientes nas dietas. O teor médio de proteína das dietas foi de $20 \%$. As determinações de matéria seca e matéria mineral para a silagem de milho e o concentrado obedeceram às recomendações da AOAC (1990).

Os animais foram levados às baias uma semana antes do início do experimento para adaptação ao local e ao bebedouro automático. Após a adaptação, os animais foram pesados e iniciou-se a etapa experimental, que teve duração de 72 dias. Esta fase foi composta por quatro períodos de 18 dias, sendo os 16 primeiros de adaptação à dieta, para evitar o efeito residual, e os últimos dois dias de colheita.

Os animais foram sorteados nos tratamentos e passaram a ser alimentados nos comedouros, na parte da manhã, com aproximadamente 3,3\% do peso corporal em MS. No fim de cada período, os animais eram pesados, para que houvesse ajuste no fornecimento

Tabela 1 - Composição percentual dos ingredientes e análise bromatológica (\%) das dietas experimentais, em base seca, e respectivos balanços cátion-aniônico das dietas em mEq/kg de MS.

\begin{tabular}{lllll}
\hline \multicolumn{1}{c}{ Ingredientes } & +150 & +250 & +400 & +500 \\
\hline Silagem de milho & 40,2 & 40,0 & 39,9 & 39,9 \\
Milho grão & 25,2 & 24,9 & 24,4 & 24,1 \\
Soja extrusada & 32,8 & 32,3 & 31,8 & 31,3 \\
Suplemento mineral & 0,6 & 0,6 & 0,6 & 0,6 \\
Calcário & 0,6 & 0,6 & 0,6 & 0,6 \\
Óxido de magnésio & 0,6 & 0,6 & 0,6 & 0,6 \\
NaHCO & 0 & 1,0 & 2,1 & 3,0 \\
$\quad$ Composição centesimal & & & 68,7 \\
Matéria seca & 68,7 & 68,6 & 68,7 & 19,2 \\
Proteína Bruta & 20,2 & 20,1 & 20,2 & 9,1 \\
Fibra Bruta & 9,4 & 9,3 & 9,2 & 13,6 \\
Extrato Etéreo & 10,7 & 10,5 & 10,3 & 9,9 \\
Matéria Mineral & 7,0 & 8,0 & 8,6 & 48,2 \\
Extrativo não nitrogenado & 52,7 & 52,1 & 51,8 & \\
\hline
\end{tabular}

Ciência Rural, v.36, n.5, set-out, 2006. 
das dietas. A água foi fornecida ad libitum, por meio dos bebedouros automáticos.

Nos dias de colheita, foram retiradas amostras de leite das duas ordenhas, para análises de gordura, acidez titulável, $\mathrm{pH}$, índice crioscópico e densidade. Essas análises foram realizadas logo após a colheita, segundo as normas analíticas do instituto ADOLFO LUTZ (1985), a saber: o pH foi determinado por meio de leitura em um pH-metro digital; a acidez, conforme o método clássico de Dornic; a determinação da densidade foi feita por termolactodensímetro de Quevenne $15^{\circ} \mathrm{C}$; o índice crioscópico foi determinado por meio de leitura digital em aparelho de crioscopia eletrônica; a gordura do leite, pelo método de Gerber.

O delineamento experimental utilizado foi o quadrado latino $(4 \mathrm{x} 4)$ replicado. Foram utilizadas oito vacas, divididas em dois quadrados latinos e quatro tratamentos. As análises estatísticas foram realizadas por meio do programa computacional SAS ${ }^{\circledR}$ versão 8.2 (SAS Institute Inc., 2000), no PROC GLM, por regressão com os níveis do BCAD (+150, +250, +400 e +500mEq $\left.\mathrm{kg}^{-1} \mathrm{MS}\right)$. Algumas variáveis também foram analisadas por meio de contrastes ortogonais, com o uso do PROC GLM. O nível de significância adotado foi 5\%.

\section{RESULTADOS E DISCUSSÃO}

Os dados referentes às porcentagens médias de gordura no leite das vacas, ao índice crioscópico e à densidade estão apresentados na tabela 2. O teor de gordura do leite não se alterou em função do BCAD $(\mathrm{P}>0,05)$. Este resultado concorda com aquele observado por WEST et al. (1992), os quais, estudando diferentes níveis de BCAD e duas fontes de cátion (Na e K) para vacas holandesas em lactação sob estresse térmico, não verificaram alterações nas concentrações de gordura do leite em função dos diferentes níveis de BCAD. Entretanto, houve aumento da gordura do leite para a fonte de Na quando comparada com a fonte de K, para um mesmo valor de BCAD. KILMER et al. (1981), ao adicionarem bicarbonato de sódio às rações de vacas, em substituição ao cloreto, conseqüentemente aumentando os valores de BCAD, não obtiveram alterações nos teores de gordura do leite. No trabalho de TUCKER et al. (1994), foi testado o efeito do sesquicarbonato de sódio (0 ou 1\%) e dois níveis de BCAD (+115 e +288mEq kg-1 MS, respectivamente) na composição de leite em vacas durante todo o período de lactação. No início e no meio da lactação, a adição de sesquicarbonato de sódio não alterou a gordura do leite. Entretanto, no final da lactação, a gordura do leite aumentou com a suplementação do sesquicarbonato. Os autores concluíram que o efeito da dieta tampão foi mais efetivo no final da lactação. Além desse resultado obtido por TUCKER et al. (1994) no final da lactação, outros autores também observaram alterações nos teores de gordura. WATERMAN et al. (1991), em experimento com vacas no meio da lactação e dietas com níveis diferentes de BCAD, observaram que a alta concentração de cátion aumentou a porcentagem de gordura do leite. WEST et al. (1991), trabalhando com vacas em lactação, relataram que a porcentagem de gordura no leite respondeu cubicamente ao aumento do BCAD. De acordo com ZIA et al. (2001), as porcentagens de gordura do leite aumentaram com o aumento do BCAD. RAMANA et al. (2002); avaliando o efeito da adição do bicarbonato de sódio na composição do leite em vacas cruzadas, encontraram aumento na gordura do leite com a adição do tampão. Em contrapartida, KENNELLY et al. (1999), estudando o efeito da inclusão de bicarbonato de sódio e de diferentes relações volumoso:concentrado em vacas holandesas, concluíram que a adição do tampão em dieta de alto concentrado diminuiu a gordura do leite. Os efeitos da adição do bicarbonato de sódio na correção de níveis baixos de gordura no leite de vacas

Tabela 2 - Valores médios de gordura (\%), índice crioscópico $\left({ }^{\circ} \mathrm{H}\right)$ e densidade $\left(\mathrm{a} 15^{\circ} \mathrm{C}\right)$ do leite de vacas alimentadas com quatro níveis de dietas catiônicas.

\begin{tabular}{|c|c|c|c|c|c|}
\hline \multicolumn{5}{|c|}{ Tratamentos (mEq/kg de MS) } & \multirow{2}{*}{$\begin{array}{c}\text { Análise de variância } \\
\text { P }\end{array}$} \\
\hline & +150 & +250 & +400 & +500 & \\
\hline Gordura & 2,640 & 2,700 & 2,690 & 2,860 & \multirow{2}{*}{0,766} \\
\hline $\mathrm{EP}^{1}$ & 0,181 & 0,159 & 0,175 & 0,256 & \\
\hline I. crioscópico & $-0,537$ & $-0,536$ & $-0,540$ & $-0,535$ & \multirow{2}{*}{0,518} \\
\hline $\mathrm{EP}^{1}$ & 0,001 & 0,002 & 0,003 & 0,002 & \\
\hline Densidade & 1,027 & 1,026 & 1,026 & 1,027 & \multirow[b]{2}{*}{0,342} \\
\hline $\mathrm{EP}^{1}$ & 0,814 & 0,716 & 0,722 & 0,851 & \\
\hline
\end{tabular}

${ }^{1}$ Erro padrão. 
alimentadas com dietas contendo baixa relação forragem/concentrado não são claros.

Os valores médios de índice crioscópico $\left(-0,537^{\circ} \mathrm{H}\right)$ e de densidade $\left(1,027 \mathrm{~g} \mathrm{~mL}^{-1}\right)$ encontrados no presente experimento foram próximos aos citados como padrão de leite cru refrigerado (IC=-0,531 ${ }^{\circ} \mathrm{H}$; $\mathrm{D}=1,028-1,034 \mathrm{~g} \mathrm{~mL}^{-1}$ ) segundo o MAPA (2002). Entretanto, as médias dos tratamentos não foram estatisticamente diferentes $(\mathrm{P}>0,05)$. Não foram encontrados na literatura trabalhos que relacionassem dietas catiônicas com índice criosópico e com densidade do leite. De acordo com BECCHI (2004), o índice crioscópico é um dos parâmetros analíticos de precisão utilizados para determinar a qualidade físicoquímica do leite, sendo um valor relacionado principalmente com a presença de maior ou menor quantidade de lactose e de cloretos no leite. Entretanto, no presente experimento, apesar de uma possível influência na concentração de cloretos no sangue, não foi observada uma relação entre a variação de BCAD e o índice crioscópico no leite. Leite com alto teor de gordura apresenta maior densidade em relação a leite com baixo teor de gordura, em razão do aumento do extrato seco desengordurado que acompanha o aumento no teor de gordura. No trabalho de WATERMAN et al. (1991), o maior BCAD aumentou a porcentagem de gordura do leite. No presente experimento, não houve diferença significativa na concentração de gordura do leite; conseqüentemente, a densidade não foi alterada.

Os dados apresentados na tabela 3 correspondem aos valores médios de $\mathrm{pH}$ e à acidez do leite. $\mathrm{O}$ pH apresentou efeito linear significativo $(P<0,01)$, em função do aumento do BCAD. A equação linear referente ao $\mathrm{pH}$ do leite em função da variação do BCAD foi a seguinte: $y=0,0002 x+6,7036$. Para a variável acidez, foi observada resposta cúbica significativa $(P<0,05)$. A equação $y=-6 E-07 x^{3}+0,0006 x^{2}$
- 0,1922x + 36,75 representa a resposta cúbica da acidez do leite em diferentes níveis de balanço cátion-aniônico da dieta. Pela análise de contrastes ortogonais, verificou-se que o tratamento com menor BCAD foi diferente $(\mathrm{P}<0,01)$ dos demais tratamentos, apresentando a maior média de acidez do leite $\left(19,13^{\circ}\right.$ D). Os tratamentos de +250 e $+400 \mathrm{mEq} \mathrm{kg}{ }^{-1} \mathrm{MS}$ não apresentaram diferença entre si $(\mathrm{P}>0,05)$. Já o tratamento com maior BCAD foi diferente significativamente $(\mathrm{P}<0,01)$ dos outros tratamentos, tendo a menor média para acidez do leite $\left(15^{\circ} \mathrm{D}\right)$.

Não foram encontrados na literatura trabalhos que relacionassem dietas catiônicas com $\mathrm{pH}$ e acidez do leite, havendo necessidade de maiores estudos sobre as propriedades físico-químicas do leite. No presente experimento, o aumento do BCAD e o conseqüente aumento da ingestão de bicarbonato de sódio pode ter proporcionado aumento do $\mathrm{pH}$ sanguíneo. CORREA (2004), em trabalho desenvolvido com vacas em lactação e níveis crescentes de BCAD, encontrou aumento linear significativo do bicarbonato e do $\mathrm{pH}$ sanguíneo. O bicarbonato é tampão tanto do sangue como do leite. Como este é um filtrado do sangue a possível passagem de bicarbonato para o leite pode ter feito com que o $\mathrm{pH}$ aumentasse, refletindo também na acidez, uma vez que, no tratamento de maior BCAD (com maior quantidade de $\mathrm{NaHCO}_{3}$ ), houve menor acidez do leite, e no tratamento de menor BCAD (sem adição de bicarbonato de sódio), esta foi maior.

\section{CONCLUSÕES}

A variação do BCAD dentro da faixa positiva não altera o teor de gordura, a crioscopia e a densidade do leite de vacas. O BCAD modifica o $\mathrm{pH}$ e a acidez do leite mesmo dentro de variação positiva. Essa modificação consiste no aumento linear do $\mathrm{pH}$ e em resposta cúbica da acidez em função do aumento do BCAD.

Tabela 3 - Níveis médios de pH e acidez ( ${ }^{\circ}$ Dornic) do leite de vacas alimentadas com quatro níveis de dietas catiônicas.

\begin{tabular}{|c|c|c|c|c|c|}
\hline & \multicolumn{4}{|c|}{ Tratamentos (mEq/kg de MS) } & \multirow{2}{*}{$\begin{array}{c}\text { Análise de variância } \\
\text { Efeito }\end{array}$} \\
\hline & +150 & +250 & +400 & +500 & \\
\hline $\mathrm{pH}$ & 6,75 & 6,76 & 6,80 & 6,83 & $\mathrm{~L}^{2}<0,01$ \\
\hline $\mathrm{EP}^{1}$ & 0,04 & 0,03 & 0,04 & 0,05 & \\
\hline Acidez & 19,13 & 16,25 & 16,63 & 15,00 & $C^{3}=0,04$ \\
\hline $\mathrm{EP}^{1}$ & 1,06 & 0,37 & 0,57 & 0,60 & \\
\hline
\end{tabular}

\footnotetext{
${ }^{1}$ Erro padrão.

${ }^{2}$ Efeito Linear.

${ }^{3}$ Efeito Cúbico.
} 


\section{REFERÊNCIAS}

AOAC. Official methods of analysis. 15.ed. Arlington: AOAC, 1990. 1298p.

BECCHI, C.S. Estudodo índice crioscópico do leite tipo B in natura produzido na bacia leiteira do Vale do Taquari, RS. Acta Scientiae Veterinariae, v.32, n.2, p.161-162, 2004.

BLOCK, E. Manipulating dietary anions and cations for prepartum dairy cows to reduce incidence of milk fever. Journal Dairy Science, Champaingn, v.67, n.12, p.29392948, 1984

CORREA, L.B. Influência de dietas catiônicas no desempenho e parâmetros ácido-base em vacas em lactação. 2004. 77f. Monografia - Faculdade de Zootecnia e Engenharia de Alimentos, Universidade de São Paulo.

KENNELLY, J.J. et al. Influence of carbohydrate source and buffer on rumen fermentation characteristics, milk yield, and milk composition in early lactation Holstein cows. Journal Dairy Science, Champaign, v.82, n.11, p.2486-2496, 1999.

KILMER, L.H. et al. Addition of sodium bicarbonate to rations of postpartum dairy cows: physiological and metabolic effects. Journal Dairy Science, Champaign, v.64, n.12, p.2357-2369, 1981.

MAPA. Ministério da Agricultura, Pecuária e Abastecimento. Instrução normativa n:51, de 18 de setembro de 2002. Acesso em 25 set. 2005. Online. Disponível na Internet em: <www.agricultura.gov.br/sda/dipoa/in51.htm>.

NORMAS ANALÍTICAS INSTITUTO ADOLFO LUTZ . Métodos físicos e químicos para análise de alimentos. 3.ed. São Paulo, 1985. 533p.
NRC, Nutrient Requeriment of Dairy Cattle. 7.ed. Washington, DC: National Academy of Sciences, 2001. 381p.

RAMANA, D.B.V. et al. Effect of feeding buffer on milk yield and composition of crossbred cows. Indian Veterinary Journal, v.79, p.53-55, 2002.

SAS. SAS Stat Guide, Release 8.2.ed. Cary, NC: SAS Institute, 2000. 1028p.

SCHNEIDER, P.L. et al. Responses of lactating cows to dietary sodium source and quantity and potassium quantity during heat stress. Journal Dairy Science, Champaign, v.69, n.1, p.99110, 1986.

TUCKER, W.B. et al. Natural sodium sesquicarbonate fed for an entire lactation: influence on performance and acid-base status of dairy cows. Journal Dairy Science, Champaign, v.77, n.10, p.531-537, 1994.

WATERMAN, D.F. et al. Role of magnesium in the dietary cation-anion balance equation for ruminants. Journal Dairy Science, Champaign, v.74, n.6, p.1866-1873, 1991.

WEST, J.W. et al. Changing dietary eletrolyte balance for dairy cows in cool and hot environments. Journal Dairy Science, v.74, p.1662-1674, 1991.

WEST, J.W. et al. Dietary cation-anion balance cation source effects on production and acid-base status of heat-stressed cows. Journal Dairy Science, Champaign, v.75, p.2776-2786, 1992.

ZIA, U. H. et al. Dietary cation-anion balance in the ruminants II - effects during mid lactation and dry period. International Journal of Agriculture and Biology, v.3, p.143-148, 2001. 